\title{
ORTHOGONAL FORMS AND ORTHOGONALITY PRESERVERS ON REAL FUNCTION ALGEBRAS REVISITED
}

\author{
ANTONIO M. PERALTA
}

\begin{abstract}
In [1] we determine the precise form of a continuous orthogonal form on a commutative real $\mathrm{C}^{*}$-algebra. We also describe the general form of a (not-necessarily continuous) orthogonality preserving linear map between commutative unital real $\mathrm{C}^{*}$-algebras. Among the consequences, we show that every orthogonality preserving linear bijection between commutative unital real $\mathrm{C}^{*}$-algebras is continuous. In this note we revisit these results and their proofs with the idea of filling a gap in the arguments, and to extend the original conclusions.
\end{abstract}

\section{INTRODUCTION}

Let $A$ be a real or complex $\mathrm{C}^{*}$-algebra. Elements $a, b$ in $A$ are said to be orthogonal (written $a \perp b$ ) if $a b^{*}=b^{*} a=0$. A bilinear form $V: A \times A \rightarrow \mathbb{C}$ is said to be orthogonal if $V(a, b)=0$ whenever $a \perp b$. In [1] we establish a generalization of a celebrated result due to S. Goldstein (see [2, Theorem 1.10]) by proving the following result:

Theorem 1.1. [1, Theorem 2.4] Let $V: A \times A \rightarrow \mathbb{R}$ be a continuous orthogonal form on a commutative real $C^{*}$-algebra, then there exist $\varphi_{1}$ and $\varphi_{2}$ in $A^{*}$ satisfying

$$
V(x, y)=\varphi_{1}(x y)+\varphi_{2}\left(x y^{*}\right),
$$

for every $x, y \in A$.

We recently realized the presence of a "gap" affecting some of the technical results given in [1]. The concrete difficulties appear in the following arguments: By the Gelfand theory for commutative real $\mathrm{C}^{*}$-algebras, every commutative unital real $\mathrm{C}^{*}$-algebra $A$ is $\mathrm{C}^{*}$-isomorphic (and hence isometric) to a real function algebra of the form $C(K)^{\tau}=\{f \in C(K): \tau(f)=f\}$, where $K$ is a compact Hausdorff space, $\tau$ is a conjugation (i.e. a conjugate linear isometry of period 2) on $C(K)$ given by $\tau(f)(t)=\overline{f(\sigma(t))}(t \in K)$, and $\sigma: K \rightarrow K$ is a topological involution (i.e. a period 2 homeomorphism) (compare [4, Proposition 5.1.4]). Let $\sigma: K \rightarrow K$ be a topological involution on a compact Hausdorff space $K$. Clearly, the sets $N=\{t \in K: \sigma(t) \neq t\}$ and $F=\{t \in K: \sigma(t)=t\}$ are open and closed subsets of $K$, respectively. It is established in [1, Lemma 2.1 and its proof] that the family, $\mathcal{F}$, of all open subsets $O \subseteq K$ such that $O \cap \sigma(O)=\emptyset$, ordered by inclusion is an inductive set, and hence, by Zorn's lemma, there exists an open subset $\mathcal{O} \subset \mathcal{F}$

2010 Mathematics Subject Classification. Primary 46H40; 4J10, Secondary 47B33; 46L40; $46 \mathrm{E} 15 ; 47 \mathrm{~B} 48$.

Key words and phrases. Orthogonal form, real $\mathrm{C}^{*}$-algebra, orthogonality preservers, disjointness preserver, separating map.

Author partially supported by the Spanish Ministry of Economy and Competitiveness project no. MTM2014-58984-P and Junta de Andalucía grant FQM375. 
maximal with respect to the property $\mathcal{O} \cap \sigma(\mathcal{O})=\emptyset$. Immediately after $[1$, Lemma 2.1 it is claimed that,

"by the maximality of $\mathcal{O}, K=F \stackrel{\circ}{\cup} \stackrel{\circ}{\cup}(\mathcal{O})$."

Unfortunately, the above equality is not always true. Consider, for example, $K=\mathbb{T}$ the unit sphere of $\mathbb{C}$ and $\sigma: K \rightarrow K, \sigma(t)=-t(t \in \mathbb{T})$. In this case $F=\emptyset$ and $\mathcal{O}=\{t \in \mathbb{T}: \Im \mathrm{m}(t)>0\}$ is a maximal set in $\mathcal{F}$, but $F \stackrel{\circ}{\cup} \cup \circ \sigma(\mathcal{O}) \neq K$. This gap affects several statements and proofs of technical results in [1, Sections 2 and 3].

We recall that a mapping $T: A \rightarrow B$ between real or complex $\mathrm{C}^{*}$-algebras is said to be orthogonality or disjointness preserving if $a \perp b$ in $A$ implies $T(a) \perp T(b)$ in $B$.

In the second main goal studied in [1], we consider orthogonality preserving linear maps between real function algebras belonging to a special subclass of the category of commutative unital real $\mathrm{C}^{*}$-algebras. The algebras in this particular subclass can be presented as follows: Let $F$ be a closed subspace of a compact Hausdorff space $K$. We denote by $C_{r}(K)=C_{r}(K ; F)$ the real $\mathrm{C}^{*}$-algebra of all continuous functions $f: K \rightarrow \mathbb{C}$ taking real values on $F$. The main result in $[1, \S 3]$ is presented in Theorem 3.2, where we establish a complete description of those linear (not necessarily continuous) orthogonality preserving operators between $C_{r}(K ; F)$ spaces. It should be remarked here that the proof of this result is not affected by the gap commented above. Among the consequences not affected by the difficulties, we obtain the following result.

Theorem 1.2. [1, Remark 3.4, proof of Theorem 3.5 and Proposition 3.6] In the notation above, let $T: C_{r}\left(K_{1} ; F_{1}\right) \rightarrow C_{r}\left(K_{2} ; F_{2}\right)$ be an orthogonality preserving linear bijection. Then $T$ is automatically continuous and $T^{-1}$ preserves invertible elements, that is, $T^{-1}(g)$ is invertible whenever $g$ is an invertible element in $C_{r}\left(L_{2}\right)$.

However, it must be remarked that the main goal expected from these results (that is, [1, Theorem 3.5]) is directly jeopardized by the mistake introduced after [1, Lemma 2.1]. The concrete obstacle appears because by applying the wrong identity commented above, we identify every commutative unital real $\mathrm{C}^{*}$-algebra with a real function algebra of the form $C_{r}(K ; F)$. More concretely, let $T: C\left(K_{1}\right)^{\tau_{1}} \rightarrow$ $C\left(K_{2}\right)^{\tau_{2}}$ be an orthogonality preserving linear mapping, and let $\sigma_{i}: K_{i} \rightarrow K_{i}$ be a topological involution satisfying $\tau_{i}(f)=\overline{f \circ \sigma_{i}}$. According to what is claimed in [1, page 287]:

"... keeping in mind the notation in the previous section, we write $L_{i}:=\mathcal{O}_{i} \cup F_{i}$, where $\mathcal{O}_{i}$ and $F_{i}=\left\{t \in K_{i}: \sigma_{i}(t)=t\right\}$ are the subsets of $K_{i}$ given by Lemma [1, Lemma 2.1]. The map sending each $f$ in $C\left(K_{i}\right)^{\tau_{i}}$ to its restriction to $L_{i}$ is a $C^{*}$ isomorphism (and hence a surjective linear isometry) from $C\left(K_{i}\right)^{\tau_{i}}$ onto the real $C^{*}$-algebra $C_{r}\left(L_{i}\right)$ of all continuous functions $f: L_{i} \rightarrow \mathbb{C}$ taking real values on $F_{i}$. Thus, studying orthogonality preserving linear maps between $C(K)^{\tau}$ spaces is equivalent to study orthogonality preserving linear mappings between the corresponding $C_{r}(L)$-spaces."

Unfortunately, since, in general, $K_{i} \neq \mathcal{O}_{i} \cup F_{i} \cup \sigma\left(\mathcal{O}_{i}\right)$, we cannot guarantee that $L_{i}:=\mathcal{O}_{i} \cup F_{i}$, is a closed subset of $K_{i}$ (compare the example given in page 2). Furthermore, there are examples of $C(K)^{\tau}$-spaces which are not real $C^{*}$-isomorphic to a real $\mathrm{C}^{*}$-algebra of the form $C_{r}(X, F)$ (compare 3.1). In summary, the proof of 
[1, Theorem 3.5] is only valid for orthogonality preserving linear bijections between commutative unital real $\mathrm{C}^{*}$-algebras which are of the form $C_{r}(K, F)$.

Though the main results in [1] remain valid in the form they are stated, the gap commented above makes invalid some of the arguments given in that paper. It is necessary to provide a complete and correct argument, which fix all the problems. We present here a complete revision of these results with new and complete arguments, which allow us to solve and fix the problems caused by the gap in the original proof. The problems caused in $[1, \S 2]$ are easily fixable and we shall just comment briefly the necessary changes in Section 2 here. However, the difficulties caused in the proof of the result asserting the automatic continuity of every orthogonality preserving linear bijection between commutative unital real $\mathrm{C}^{*}$-algebras force us to present a more detailed revision in Section 3.

\section{Orthogonal forms on REAL C*-Algebras}

We revise in this section the proof of [1, Theorem 2.4]. For conciseness reasons, we keep the notation in [1] without inserting explicit definitions. We shall confine ourselves to state the minimum changes necessary to fix the difficulties in $[1, \S 2]$.

Lemma 2.2 in [1] should be rewritten as follows:

Lemma 2.2. In the notation of Lemma 2.1, let $B(A)=B(K)^{\tau}$, let $a \in B(K)_{s a}^{\tau}$,

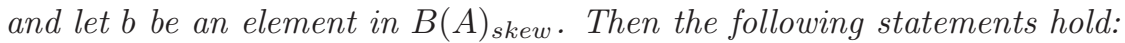

a) $b \mid F=0$;

b) For each $\varepsilon>0$, there exist mutually disjoint Borel sets $B_{1}, \ldots, B_{m}$ and real numbers $\lambda_{1}, \ldots, \lambda_{m}$ satisfying $\sigma\left(B_{j}\right) \cap B_{j}=\emptyset$ and $\left\|b-\sum_{j=1}^{m} i \lambda_{j}\left(\chi_{B_{j}}-\chi_{\sigma\left(B_{j}\right)}\right)\right\|<\varepsilon$;

c) For each $\varepsilon>0$, there exist mutually disjoint Borel sets $C_{1}, \ldots, C_{m} \subset K$ and real numbers $\mu_{1}, \ldots, \mu_{m}$ satisfying $\sigma\left(C_{j}\right)=C_{j}$ and $\left\|a-\sum_{j=1}^{m} \mu_{j} \chi_{C_{j}}\right\|<\varepsilon$.

The arguments given in [1, Comments before Lemma 2.1 and Proof of Lemma 2.2] remain valid here.

The notation in [1, Lemma 2.3] should be replaced with the following: For each $C \subseteq K$ with $C \cap \sigma(C)=\emptyset$ we shall write $u_{C}=i\left(\chi_{C}-\chi_{\sigma(C)}\right)$. The symbol $u_{0}$ will stand for the projection $\chi_{K \backslash F}$. Clearly, $1=\chi_{F}+u_{0}$ where 1 is the unit element in $B(K)^{\tau}$. By Lemma $2.2 a$ ), for each $b \in B(K)_{\text {skew }}^{\tau}$ we have $b \perp \chi_{F}$, and so $b=b u_{0}$. The statement of [1, Proposition 2.3] should be modified in the following sense:

Proposition 2.3. Let $K$ be a compact Hausdorff space, $\tau$ a period-2 conjugatelinear isometric *-homomorphism on $C(K), A=C(K)^{\tau}$, and $V: A \times A \rightarrow \mathbb{R}$ be an orthogonal bounded bilinear form whose Arens extension is denoted by $V^{* *}$ : $A^{* *} \times A^{* *} \rightarrow \mathbb{R}$. Let $\sigma: K \rightarrow K$ be a period-2 homeomorphism satisfying $\tau(a)(t)=$ $\overline{a(\sigma(t))}$, for all $t \in K, a \in C(K)$. Then the following assertions hold for all Borel subsets $D, B, C$ of $K$ with $\sigma(B) \cap B=\sigma(C) \cap C=\emptyset$ and $\sigma(D)=D$ :

a) $V\left(\chi_{D}, u_{B}\right)=V\left(u_{B}, \chi_{D}\right)=0$, whenever $D \cap B=\emptyset$;

b) $V\left(u_{B}, u_{C}\right)=0$, whenever $B \cap C=\emptyset$;

c) $V\left(\left(u_{0}-u_{C} u_{C}^{*}\right) u_{B}, u_{C}\right)=V\left(u_{C},\left(u_{0}-u_{C} u_{C}^{*}\right) u_{B}\right)=0$. 
The proof given in [1] remains valid with the obvious changes in the notation. We include here an sketch of the changes for completeness reasons.

Proof. By an abuse of notation, we identify $V$ and $V^{* *}$. Arguing as in the first part of the proof of [1, Proposition 2.3] we get

$$
V\left(u_{K_{2}}, \frac{1}{2}\left(\chi_{K_{1}}+\chi_{\sigma\left(K_{1}\right)}\right)\right)=V\left(\frac{1}{2}\left(\chi_{K_{1}}+\chi_{\sigma\left(K_{1}\right)}\right), u_{K_{2}}\right)=0
$$

and

$$
V\left(u_{K_{1}}, u_{K_{2}}\right)=0,
$$

whenever $K_{1}$ and $K_{2}$ are two compact subsets of $K$ such that $K_{1}, K_{2}, \sigma\left(K_{1}\right)$ and $\sigma\left(K_{2}\right)$ are pairwise disjoint.

a) Let now $D, B$ be two disjoint Borel subsets of $K$ such that $\sigma(D)=D$ and $B \cap \sigma(B)=\emptyset$. By inner regularity there exist nets of the form $\left(\chi_{K_{\lambda}^{D}}\right)_{\lambda}$ and $\left(\chi_{K_{\gamma}^{B}}\right)_{\gamma}$ such that $\left(\chi_{K_{\lambda}^{D}}\right)_{\lambda}$ and $\left(\chi_{K_{\gamma}^{B}}\right)_{\gamma}$ converge in the weak* topology of $C(K)^{* *}$ to $\chi_{D}$ and $\chi_{B}$, respectively, where each $K_{\lambda}^{D} \subseteq D$ and each $K_{\gamma}^{B} \subseteq B$ is a compact subset of $K$. By the assumptions made on $D$ and $B$ we have that $K_{\lambda}^{D} \cap K_{\gamma}^{B}=K_{\lambda}^{D} \cap \sigma\left(K_{\gamma}^{B}\right)=\emptyset$ and $K_{\gamma}^{B} \cap \sigma\left(K_{\gamma}^{B}\right)=\emptyset$ for all $\lambda$ and $\gamma$. By (1) and the separate weak* continuity of $V$ we have

$$
V\left(\chi_{D}, u_{B}\right)=w^{*}-\lim _{\lambda}\left(w^{*}-\lim _{\gamma} V\left(\frac{\chi_{K_{\lambda}^{D}}+\chi_{\sigma\left(K_{\lambda}^{D}\right)}}{2}, u_{K_{\gamma}^{B}}\right)\right)=0,
$$

and

$$
V\left(u_{B}, \chi_{D}\right)=0
$$

A similar argument, but replacing (1) with (2), applies to obtain $b$ ).

To prove the last statement, we observe that

$$
\left(u_{0}-u_{c} u_{C}^{*}\right) u_{B}=\left(\chi_{K \backslash F}-\chi_{C}-\chi_{\sigma(C)}\right) u_{B}=\chi_{K \backslash(F \cup C \cup \sigma(C))} u_{B}=u_{(K \backslash(C \cup \sigma(C))) \cap B},
$$

and hence the statement $c$ ) follows from $b$ ).

The statement of Theorem 2.4 in [1] remains unaltered, however, the proof of this theorem needs a slight modification from line 11.

Proof of Theorem 1.1. By Proposition [1, Proposition 1.5] we have

$$
V\left(a_{1}, a_{2}\right)=V\left(a_{1} a_{2}, 1\right),
$$

for every $a_{1}, a_{2}$ in $B(K)_{s a}^{\tau}$.

To deal with the skew-symmetric part, let $D, B, C$ be Borel subsets of $K$ with, $D=\sigma(D), B \cap \sigma(B)=\emptyset$ and $C \cap \sigma(C)=\emptyset$. From Proposition $2.3 a)$, we have

$$
\begin{gathered}
V\left(\chi_{D}, u_{B}\right)=V\left(\chi_{D}, u_{B}\left(1-\chi_{D}+\chi_{D}\right)\right)=V\left(\chi_{D}, u_{B \cap(K \backslash D)}\right)+V\left(\chi_{D}, u_{B} \chi_{D}\right) \\
=V\left(\chi_{D}-1+1, u_{B} \chi_{D}\right)=V\left(-\chi_{(K \backslash D)}+1, u_{(B \cap D)}\right)=V\left(1, u_{B} \chi_{D}\right),
\end{gathered}
$$

and similarly,

$$
V\left(u_{B}, \chi_{D}\right)=V\left(u_{B} \chi_{D}, 1\right)
$$


If we apply Proposition $2.3 b$ ) and $c$ ), repeatedly, we deduce that

$$
\begin{gathered}
V\left(u_{B}, u_{C}\right)=V\left(u_{B} u_{0}, u_{C}\right)=V\left(u_{B}\left(u_{0}+u_{C} u_{C}^{*}-u_{C} u_{C}^{*}\right), u_{C}\right) \\
=V\left(u_{B} u_{C} u_{C}^{*}, u_{C}\right)=V\left(u_{B} u_{C} u_{C}^{*}, u_{C}-u_{0}+u_{0}\right) \\
=V\left(u_{(B \cap C)},-u_{((K \backslash F) \backslash C)}+u_{0}\right)=V\left(u_{(B \cap C)}, u_{0}\right)=V\left(u_{B} u_{C}, u_{0}\right) .
\end{gathered}
$$

and similarly

$$
V\left(u_{B}, u_{C}\right)=V\left(u_{0}, u_{B} u_{C}\right) .
$$

Let $a_{l}=\sum_{j=1}^{m_{l}} \mu_{l, j} \chi_{D_{j}^{l}}, b_{l}=\sum_{k=1}^{p_{l}} \lambda_{l, k} u_{B_{k}^{l}}(l \in\{1,2\})$ be two simple elements in $B(K)_{s a}^{\tau}$ and $B(K)_{\text {skew }}^{\tau}$, respectively, where $\lambda_{l, k}, \mu_{l, j} \in \mathbb{R}$, for each $l \in\{1,2\}$, $\left\{D_{1}^{l}, \ldots, D_{m_{l}}^{l}\right\}$ and $\left\{B_{1}^{l}, \ldots, B_{p_{l}}^{l}\right\}$ are families of mutually disjoint Borel subsets of $K$ with $\sigma\left(D_{j}^{l}\right)=D_{j}^{l}$ and $B_{i}^{l} \cap \sigma\left(B_{i}^{l}\right)=\emptyset$. By (5), (6), (7), and (8), we have

$$
\begin{gathered}
V\left(a_{1}+b_{1}, a_{2}+b_{2}\right)=V\left(a_{1} a_{2}, 1\right)+\sum_{j=1}^{m_{1}} \sum_{k=1}^{p_{2}} \mu_{1, j} \lambda_{2, k} V\left(\chi_{D_{j}^{1}}, u_{B_{k}^{2}}\right) \\
+\sum_{k=1}^{p_{1}} \sum_{j=1}^{m_{2}} \mu_{2, j} \lambda_{1, k} V\left(u_{B_{k}^{1}}, \chi_{D_{j}^{2}}\right)+\sum_{k=1}^{p_{1}} \sum_{k=1}^{p_{2}} \lambda_{2, k} \lambda_{1, k} V\left(u_{B_{k}^{1}}, u_{B_{k}^{2}}\right) \\
=V\left(a_{1} a_{2}, 1\right)+\sum_{j=1}^{m_{1}} \sum_{k=1}^{p_{2}} \mu_{1, j} \lambda_{2, k} V\left(1, \chi_{D_{j}^{1}} u_{B_{k}^{2}}\right) \\
+\sum_{k=1}^{p_{1}} \sum_{j=1}^{m_{2}} \mu_{2, j} \lambda_{1, k} V\left(u_{B_{k}^{1}} \chi_{D_{j}^{2}}, 1\right)+\sum_{k=1}^{p_{1}} \sum_{k=1}^{p_{2}} \lambda_{2, k} \lambda_{1, k} V\left(u_{B_{k}^{1}} u_{B_{k}^{2}}, u_{0}\right) \\
=V\left(a_{1} a_{2}, 1\right)+V\left(1, a_{1} b_{2}\right)+V\left(b_{1} a_{2}, 1\right)+V\left(b_{1} b_{2}, u_{0}\right) \\
=\psi_{1}\left(a_{1} a_{2}\right)+\psi_{2}\left(a_{1} b_{2}\right)+\psi_{1}\left(b_{1} a_{2}\right)+\psi_{4}\left(b_{1} b_{2}\right),
\end{gathered}
$$

where $\psi_{1}, \psi_{2}$, and $\psi_{4}$ are the functionals in $A^{*}$ defined by $\psi_{1}(x)=V(x, 1), \psi_{2}(x)=$ $V(1, x)$, and $\psi_{4}(x)=V\left(x, u_{0}\right)$, respectively. Since, by Lemma 2.2, simple elements of the above form are norm-dense in $B(K)_{s a}^{\tau}$ and $B(K)_{\text {skew }}^{\tau}$, respectively, and $V$ is continuous, we deduce that

$$
V\left(a_{1}+b_{1}, a_{2}+b_{2}\right)=\psi_{1}\left(a_{1} a_{2}\right)+\psi_{2}\left(a_{1} b_{2}\right)+\psi_{1}\left(b_{1} a_{2}\right)+\psi_{4}\left(b_{1} b_{2}\right),
$$

for every $a_{1}, a_{2} \in B(K)_{\text {sa }}^{\tau}, b_{1}, b_{2} \in B(K)_{\text {skew }}^{\tau}$.

Now, taking $\phi_{1}=\frac{1}{4}\left(2 \psi_{1}+\psi_{2}+\psi_{4}\right), \phi_{2}=\frac{1}{4}\left(2 \psi_{1}-\psi_{2}-\psi_{4}\right), \phi_{3}=\frac{1}{4}\left(\psi_{2}-\psi_{4}\right)$, and $\phi_{4}=\frac{1}{4}\left(\psi_{4}-\psi_{2}\right)$, we get

$$
\begin{gathered}
V\left(a_{1}+b_{1}, a_{2}+b_{2}\right)=\phi_{1}\left(\left(a_{1}+b_{1}\right)\left(a_{2}+b_{2}\right)\right)+\phi_{2}\left(\left(a_{1}+b_{1}\right)\left(a_{2}+b_{2}\right)^{*}\right) \\
+\phi_{3}\left(\left(a_{1}+b_{1}\right)^{*}\left(a_{2}+b_{2}\right)\right)+\phi_{4}\left(\left(a_{1}+b_{1}\right)^{*}\left(a_{2}+b_{2}\right)^{*}\right),
\end{gathered}
$$

for every $a_{1}, a_{2} \in B(K)_{s a}^{\tau}, b_{1}, b_{2} \in B(K)_{\text {skew }}^{\tau}$.

Finally, defining $\varphi_{1}(x)=\phi_{1}(x)+\phi_{4}\left(x^{*}\right)$ and $\varphi_{2}(x)=\phi_{2}(x)+\phi_{3}\left(x^{*}\right)(x \in A)$, we get the desired statement. 


\section{Orthogonality preservers Between $C(K)^{\tau}$-Spaces}

In this section we shall study orthogonality preserving linear bijections between commutative unital real $\mathrm{C}^{*}$-algebras. The aim is to provide to the reader an argument to avoid the difficulties in the proof of [1, Theorem 3.5]. We have already commented in the introduction that the arguments in the proof of [1, Theorem 3.5] are only valid to show that every orthogonality preserving linear bijection between $C_{r}(K ; F)$-spaces is continuous (compare also page 287 and section 3 in the same paper).

We begin this section with a remark that present a commutative unital real $\mathrm{C}^{*}$ algebra which is not $\mathrm{C}^{*}$-isomorphic to a real function algebra of the form $C_{r}(K, F)$.

Remark 3.1. Let $K=\left\{t_{1}, t_{2}\right\}$ equipped with the discrete topology, $\sigma: K \rightarrow K$ the topological involution given by $\sigma\left(t_{1}\right)=t_{2}$. It is easy to check that $C(K)^{\tau} \equiv \mathbb{C}_{\mathbb{R}}$, the complex field regarded as a real space. Suppose there exists a compact Hausdorff space $X$ and a closed subset $F \subseteq X$ such that $C(K)^{\tau} \equiv \mathbb{C}_{\mathbb{R}}$ is $\mathrm{C}^{*}$-isomorphic to $C_{r}(X ; F)$. Since $C(X, \mathbb{R})$ is a real subspace of $C_{r}(X ; F)$, we easily deduce from Urysohn's lemma that $\sharp X \leq 2$ and hence $X=\left\{s_{1}, s_{2}\right\}$. In this case, there are only three possibilities to consider, namely, $F=\emptyset, F=\left\{s_{2}\right\}$ and $F=X$. The real $\mathrm{C}^{*}$-algebra $C_{r}(X, F)$ coincides with $C(K)=\mathbb{C} \oplus^{\infty} \mathbb{C}, \mathbb{C} \oplus^{\infty} \mathbb{R}$ and $\mathbb{R} \oplus^{\infty} \mathbb{R}$, respectively. None of the above real $\mathrm{C}^{*}$-algebras is $\mathrm{C}^{*}$-isomorphic to $C(K)^{\tau} \equiv \mathbb{C}_{\mathbb{R}}$.

The above Remark 3.1 implies that we cannot derived that every orthogonality preserving linear bijection between commutative unital real $\mathrm{C}^{*}$-algebras is (automatically) continuous as a consequence of [1, Theorem 3.2, Remark 3.4 and the proof of Theorem 3.5].

Henceforth, let $T: C\left(K_{1}\right)^{\tau_{1}} \rightarrow C\left(K_{2}\right)^{\tau_{2}}$ be an orthogonality preserving real linear bijection. Following standard notation, for each $s \in K_{2}$, we denote by $\delta_{s}$ : $C\left(K_{2}\right)^{\tau_{2}} \rightarrow \mathbb{C}$ the linear mapping given by $\delta_{s}(g)=g(s)\left(g \in C\left(K_{2}\right)^{\tau_{2}}\right)$. We observe that $T$ being surjective implies that $\delta_{s} T: C\left(K_{1}\right)^{\tau_{1}} \rightarrow \mathbb{C}$ is a non-zero linear map. The symbol $\operatorname{supp}\left(\delta_{s} T\right)$ will denote the set of all $t \in K_{1}$ such that for each open set $U=\sigma_{1}(U) \subseteq K_{1}$ with $t \in U$ there exists $f \in C\left(K_{1}\right)^{\tau_{1}}$ with $\operatorname{coz}(f) \subseteq U$ and $\delta_{s}(T(f)) \neq 0$. Let us recall that the cozero set, $\operatorname{coz}(f)$, of a function $f \in C\left(K_{1}\right)^{\tau_{1}}$ is the set $\left\{t \in K_{1}: f(t) \neq 0\right\}$. The equality $\sigma_{1}(\operatorname{coz}(f))=\operatorname{coz}(f)$ holds for every $f \in C\left(K_{1}\right)^{\tau_{1}}$.

Proposition 3.2. (a) For each $s \in K_{2}$ there exists a unique element $t_{s} \in K_{1}$ such that the set $\operatorname{supp}\left(\delta_{s} T\right)=\left\{t_{s}, \sigma_{1}\left(t_{s}\right)\right\}$;

(b) For every $s \in K_{2}$, we have $\delta_{s} T$ is continuous if and only if $\delta_{\sigma_{2}(s)} T$ is continuous. Moreover, the equality $\operatorname{supp}\left(\delta_{s} T\right)=\operatorname{supp}\left(\delta_{\sigma_{2}(s)} T\right)$ holds for every $s \in K_{2}$.

Proof. a) Let us take $s \in K_{2}$. We first show that $\operatorname{supp}\left(\delta_{s} T\right)$ contains at most two points of the form $t$ and $\sigma_{1}(t)$. Arguing by contradiction, we assume that there exist $t_{1}, t_{2}$ in $\operatorname{supp}\left(\delta_{s} T\right)$ with $t_{1} \neq t_{2}, \sigma_{1}\left(t_{2}\right)$. In this case, we can find two open disjoint subsets $U_{1}$ and $U_{2}$ in $K_{1}$ with $t_{i} \in U_{i}=\sigma_{i}\left(U_{i}\right)$, and two elements $f_{1}, f_{2} \in C\left(K_{1}\right)^{\tau_{1}}$ satisfying $\operatorname{coz}\left(f_{i}\right) \subseteq U_{i}$ and $\delta_{s} T\left(f_{i}\right) \neq 0$, for every $i=1,2$. This is impossible because $f_{1} \perp f_{2}$ and $T$ is orthogonality preserving.

We shall show next that $\operatorname{supp}\left(\delta_{s} T\right) \neq \emptyset$. Otherwise, $\operatorname{supp}\left(\delta_{s} T\right)=\emptyset$. Then, for each $t \in K_{1}$, there exists an open subset $U_{t}=\sigma_{1}\left(U_{t}\right)$ with $t \in U_{t}$ and $\delta_{s}(T(f))=$ 0 for every $f \in C\left(K_{1}\right)^{\tau_{1}}$ with $\operatorname{coz}(f) \subseteq U_{t}$. By a compactness argument, we can find a finite open cover $\left\{U_{1}, \ldots, U_{m}\right\}$ of $K_{1}$ satisfying $U_{k}=\sigma_{1}\left(U_{k}\right)(\forall k)$ and 
$\delta_{s}(T(f))=0$ for every $f \in C\left(K_{1}\right)^{\tau_{1}}$ with $\operatorname{coz}(f) \subseteq U_{k}$ for some $k$. Let $g_{1}, \ldots, g_{m}$ be a continuous decomposition of the identity in $C\left(K_{1}\right)$ subordinate to $U_{1}, \ldots, U_{m}$. Since $U_{k}=\sigma_{1}\left(U_{k}\right)$, the elements $f_{1}=\frac{g_{1}+\tau_{1}\left(g_{1}\right)}{2}, \ldots, f_{m}=\frac{g_{m}+\tau_{1}\left(g_{m}\right)}{2}$ define a continuous decomposition of the unit in $C\left(K_{1}^{2}\right)^{\tau_{1}}$ subordinate to $U_{1}^{2}, \ldots, U_{m}$. For each $f \in C\left(K_{1}\right)^{\tau_{1}}$ we have

$$
\delta_{s} T(f)=\sum_{k=1}^{m} \delta_{s} T\left(f f_{i}\right)=0
$$

which contradicts $\delta_{s} T \neq 0$.

b) Let $s \in K_{2}$. Clearly, $\delta_{s}(g)=\overline{\delta_{\sigma_{2}(s)}(g)}$, for every $g \in C\left(K_{2}\right)^{\tau_{2}}$. The first statement follows from the identity $\delta_{s} T=\overline{\delta_{\sigma_{2}(s)} T}$. Let us assume that $\operatorname{supp}\left(\delta_{s} T\right) \neq$ $\operatorname{supp}\left(\delta_{\sigma_{2}(s)} T\right)$. In this case there exist $t_{1} \in \operatorname{supp}\left(\delta_{s} T\right)$ and $t_{2} \in \operatorname{supp}\left(\delta_{\sigma_{2}(s)} T\right)$ with $t_{1} \neq t_{2}, \sigma_{1}\left(t_{2}\right)$. We can find two open disjoint subsets $U_{1}$ and $U_{2}$ satisfying $t_{i} \in$ $U_{i}=\sigma_{1}\left(U_{i}\right)$ and two elements $f_{1}, f_{2} \in C\left(K_{1}\right)^{\tau_{1}}$ with $\operatorname{coz}\left(f_{i}\right) \subseteq U_{i}, \delta_{s} T\left(f_{1}\right) \neq 0$ and $\delta_{\sigma_{2}(s)} T\left(f_{2}\right)=\overline{\delta_{s} T\left(f_{2}\right)} \neq 0$, which contradicts $T\left(f_{1}\right) \perp T\left(f_{2}\right)$.

Let us define an equivalence relation on $K_{i}$ given by $t \sim s$ if $\sigma_{i}(t)=\sigma_{i}(s)$. It is known that the quotient space $\left[K_{i}\right]=K_{i} / \sim$ is compact. It is not hard to check that $\left[K_{i}\right]=K_{i} / \sim$ is a compact Hausdorff space. The equivalence class of an element $t \in K_{i}$ is denoted by $[t]=\left\{t^{\prime} \in K_{i}: t^{\prime} \sim t\right\}$. Applying Proposition $3.2(a)$, we can define a map $\varphi: K_{2} \rightarrow\left[K_{1}\right], s \mapsto[t]=\operatorname{supp}\left(\delta_{s} T\right)$. By Proposition $3.2(b), \operatorname{supp}\left(\delta_{s_{1}} T\right)=\operatorname{supp}\left(\delta_{s_{2}} T\right)$, for every $s_{1}, s_{2}$ in $K_{2}$ with $s_{1} \sim s_{2}$. Therefore, the mapping $[\varphi]:\left[K_{2}\right] \rightarrow\left[K_{1}\right],[s] \mapsto[t]=\operatorname{supp}\left(\delta_{s} T\right)$ is well defined.

Lemma 3.3. Let $s$ be an element in $K_{2}$, then $\delta_{s} T(f)=0$ for every $f \in C\left(K_{1}\right)^{\tau_{1}}$ with $\operatorname{supp}\left(\delta_{s} T\right) \cap \overline{\operatorname{coz}(f)}=\emptyset$. In particular, the set $\mathcal{S} u p p\left(K_{2}\right)=\bigcup_{s \in K_{2}} \operatorname{supp}\left(\delta_{s} T\right)$ is dense in $K_{1}$.

Proof. Suppose we have $f \in C\left(K_{1}\right)^{\tau_{1}}$ with $\operatorname{supp}\left(\delta_{s} T\right) \cap \overline{\operatorname{coz}(f)}=\emptyset$. We can find an open set $U \subseteq K_{1}$ with $\operatorname{supp}\left(\delta_{s} T\right) \subseteq U=\sigma_{1}(U)$ and $U \cap \overline{\operatorname{coz}(f)}=\emptyset$. By assumptions, there exists $g \in C\left(K_{1}\right)^{\tau_{1}}$ with $\operatorname{coz}(g) \subset U$ and $\delta_{s} T(g) \neq 0$. T being orthogonality preserving and $f \perp g$ imply that $T(f) \perp T(g)$, and hence $\delta_{s} T(f)=0$.

For the second statement, suppose we can find $t_{0} \in K_{1} \backslash \overline{\left(\bigcup_{s \in K_{2}} \operatorname{supp}\left(\delta_{s} T\right)\right)}$. We observe that $\bigcup_{s \in K_{2}} \operatorname{supp}\left(\delta_{s} T\right)$ is $\sigma_{1}$-symmetric. Then, by Urysohn's lemma, there exists $f_{0} \in C\left(K_{1}\right)^{\tau_{1}}$ such that $0 \leq f_{0} \leq 1, f_{0}\left(t_{0}\right)=1$ and $\operatorname{coz}\left(f_{0}\right) \cap \overline{\bigcup_{s \in K_{2}} \operatorname{supp}\left(\delta_{s} T\right)}=$ $\emptyset$. We deduce from the first part of the lemma that $T\left(f_{0}\right)(s)=0$, for every $s \in K_{2}$, which contradicts the injectivity of $T$.

We shall derive next some consequences of the previous results.

Proposition 3.4. Let $s$ be an element in $K_{2}$ such that $\delta_{s} T: C\left(K_{1}\right)^{\tau_{1}} \rightarrow \mathbb{C}$ is a continuous linear map. Then, for each $t \in[\varphi][s]=\operatorname{supp}\left(\delta_{s} T\right)$, there exist $\lambda_{s}, \mu_{s} \in \mathbb{C}$ such that

$$
\delta_{s} T(f)=\lambda_{s} \Re e \delta_{t}(f)+\mu_{s} \Im m \delta_{t}(f),
$$


for every $f \in C\left(K_{1}\right)^{\tau_{1}}$. Moreover, $\lambda_{s}$ is unique for every $s$, while $\mu_{s}$ is unique whenever $\operatorname{supp}\left(\delta_{s} T\right)$ contains two points (i.e. $\left.\sigma_{1}\left(t_{s}\right) \neq t_{s}\right)$. It is also clear that $\lambda_{s}=T(1)(s)$, for every $s$ as above.

Proof. We already know that $\operatorname{supp}\left(\delta_{s} T\right)=\left\{t_{s}, \sigma_{1}\left(t_{s}\right)\right\}$, for a unique $t_{s} \in K_{1}$. We fix this $t_{s}$.

Let us consider the sets $J_{\text {supp }\left(\delta_{s} T\right)}:=\left\{f \in C\left(K_{1}\right)^{\tau_{1}}: \operatorname{supp}\left(\delta_{s} T\right) \cap \overline{\operatorname{coz}(f)}=\emptyset\right\}$ and $K_{\text {supp }\left(\delta_{s} T\right)}:=\left\{f \in C\left(K_{1}\right)^{\tau_{1}}:\left.f\right|_{\operatorname{supp}\left(\delta_{s} T\right)}=0\right\}$. Clearly, $J_{\text {supp }\left(\delta_{s} T\right)} \subseteq K_{\text {supp }\left(\delta_{s} T\right)}$. The arguments given by K. Jarosz in $[3,141]$ remain valid to show that $J_{\text {supp }\left(\delta_{s} T\right)}$ is normdense in $K_{\text {supp }\left(\delta_{s} T\right)}$. Lemma 3.3 implies that $J_{\text {supp }\left(\delta_{s} T\right)} \subseteq \operatorname{ker}\left(\delta_{s} T\right)=\operatorname{ker}\left(\delta_{\sigma_{2}(s)} T\right)$. We deduce from the continuity of $\delta_{s} T$ that

$$
\operatorname{ker}\left(\delta_{t_{s}}\right)=\operatorname{ker}\left(\delta_{\sigma_{1}\left(t_{s}\right)}\right)=K_{\text {supp }\left(\delta_{s} T\right)} \subseteq \operatorname{ker}\left(\delta_{s} T\right)=\operatorname{ker}\left(\delta_{\sigma_{2}(s)} T\right) .
$$

The real linear functionals $\Re \mathrm{e} \delta_{s} T, \Im \mathrm{m} \delta_{s} T, \Re \mathrm{e} \delta_{t_{s}}$, and $\Im \mathrm{m} \delta_{t_{s}}$ are all continuous. Since $\operatorname{ker}\left(\Re \mathrm{e} \delta_{t_{s}}\right) \cap \operatorname{ker}\left(\Im \mathrm{m} \delta_{t_{s}}\right)=\operatorname{ker}\left(\delta_{t_{s}}\right)$ and $\operatorname{ker}\left(\delta_{s} T\right)=\operatorname{ker}\left(\Re \mathrm{e} \delta_{s} T\right) \cap \operatorname{ker}\left(\Im \mathrm{m} \delta_{s} T\right)$, we deduce from (10) the existence of $\alpha_{1}, \alpha_{2}, \beta_{1}, \beta_{2} \in \mathbb{R}$ satisfying

$$
\Re \mathrm{e} \delta_{s} T=\alpha_{1} \Re \mathrm{e} \delta_{t_{s}}+\alpha_{2} \Im \mathrm{m} \delta_{t_{s}}
$$

and

$$
\Im \mathrm{m} \delta_{s} T=\beta_{1} \Re \mathrm{e} \delta_{t_{s}}+\beta_{2} \Im \mathrm{m} \delta_{t_{s}} .
$$

Taking $\lambda_{s}=\alpha_{1}+i \beta_{1}$ and $\mu_{s}=\alpha_{2}+i \beta_{2}$ we obtain

$$
\delta_{s} T=\lambda_{s} \Re \mathrm{e} \delta_{t_{s}}+\mu_{s} \Im \mathrm{m} \delta_{t_{s}} .
$$

To prove the second statement suppose there are $\lambda_{1}, \lambda_{2}, \mu_{1}$ and $\mu_{2}$ in $\mathbb{C}$ such that

$$
\lambda_{2} \Re \mathrm{e} \delta_{t_{s}}+\mu_{2} \Im \mathrm{m} \delta_{t_{s}}=\delta_{s} T=\lambda_{1} \Re \mathrm{e} \delta_{t_{s}}+\mu_{1} \Im \mathrm{m} \delta_{t_{s}} .
$$

Pick, via Urysohn's lemma, a function $f_{0} \in C\left(K_{1}\right)^{\tau_{1}}$ satisfying $f_{0}\left(t_{s}\right)=1$. Then $\lambda_{1}=\lambda_{2}$. When $\sigma\left(t_{s}\right) \neq t_{s}$, we can find another function $f_{1} \in C\left(K_{1}\right)^{\tau_{1}}$ satisfying $f_{1}\left(t_{s}\right)=i$, and hence $\mu_{1}=\mu_{2}$.

Corollary 3.5. Under the above conditions, the following statements hold:

(a) Let $s$ be an element in $K_{2}$ such that $\delta_{s} T: C\left(K_{1}\right)^{\tau_{1}} \rightarrow \mathbb{C}$ is a continuous linear map and $\sigma_{2}(s) \neq s$. Then $f\left(t_{s}\right)=0$, for every $t_{s} \in \operatorname{supp}\left(\delta_{s} T\right)$;

(b) Suppose $s_{1}, s_{2}$ are elements in $K_{2}$ such that $\sigma_{2}\left(s_{j}\right) \neq s_{j}$ for every $j=1,2$, $\delta_{s_{1}} T$ and $\delta_{s_{2}} T$ are continuous. If $\operatorname{supp}\left(\delta_{s_{1}} T\right)=\operatorname{supp}\left(\delta_{s_{2}} T\right)$ then $s_{1}=s_{2}$ or $s_{1}=\sigma_{2}\left(s_{2}\right)$.

Proof. (a) Let us fix $t_{s} \in \operatorname{supp}\left(\delta_{s} T\right)$. By Proposition 3.4 there exist $\lambda_{s}, \mu_{s} \in \mathbb{C}$ such that

$$
\delta_{s} T(f)=\lambda_{s} \Re \mathrm{e} \delta_{t_{s}}(f)+\mu_{s} \Im \mathrm{m} \delta_{t_{s}}(f),
$$

for every $f \in C\left(K_{1}\right)^{\tau_{1}}$. The surjectivity of $T$ implies that for each $\omega \in \mathbb{C}$ there are real numbers $\alpha, \beta$ such that $\omega=\lambda_{s} \alpha+\mu_{s} \beta$. This proves that $\left\{\lambda_{s}, \mu_{s}\right\}$ is a basis of the real space $\mathbb{C}_{\mathbb{R}}$. Therefore, by (11), $\delta_{s} T(f)=0$ implies $\Re \operatorname{e} f\left(t_{s}\right)=\Im \mathrm{m} f\left(t_{s}\right)=$ $\delta_{t_{s}}(f)=f\left(t_{s}\right)=0$, and also $f\left(\sigma_{1}\left(t_{s}\right)\right)=0$.

(b) Let us assume that $\operatorname{supp}\left(\delta_{s_{1}} T\right)=\operatorname{supp}\left(\delta_{s_{2}} T\right)$ for $s_{1}$ and $s_{2}$ as in the hypothesis. By $(a), \delta_{s_{1}} T(f)=0$ implies $f\left(t_{s}\right)=0$, for every $t_{s} \in \operatorname{supp}\left(\delta_{s_{1}} T\right)$, which, from (11), entails that $\delta_{s_{2}} T(f)=0$. Since $C\left(K_{2}\right)^{\tau_{2}}$ separates points $s_{1}, s_{2}$ in $K_{2}$ with $s_{1} \nsim s_{2}$, the surjectivity of $T$ gives $s_{1} \sim s_{2}$. 
Given $s \in K_{2}$ we denote by $\left\|\delta_{s} T\right\|$ the norm of the linear mapping $\delta_{s} T$ : $C\left(K_{1}\right)^{\tau_{1}} \rightarrow \mathbb{C}$ if the latter map is continuous, we set $\left\|\delta_{s} T\right\|=\infty$ otherwise. By Proposition 3.2(b), $\left\|\delta_{s} T\right\|=\left\|\delta_{\sigma(s)} T\right\|$, for every $s \in K_{2}$.

Lemma 3.6. Let $s \in K_{2}$ and $t \in K_{1}$ such that $[t]=\operatorname{supp}\left(\delta_{s} T\right)$. Let $U=\sigma_{1}(U) \subset$ $K_{1}$ be an open set satisfying $[t] \subset U$. The following statements hold:

(a) If $\left\|\delta_{s} T\right\|<\infty$, then for each $\varepsilon>0$ there exists $f \in C\left(K_{1}\right)^{\tau_{1}}$ such that $\|f\| \leq 1$, $\operatorname{coz}(f) \subset U$ and $\left|\delta_{s} T(f)\right|>\left\|\delta_{s} T\right\|-\varepsilon$;

(b) If $\left\|\delta_{s} T\right\|=\infty$, then for each $R>0$ there exists $f \in C\left(K_{1}\right)^{\tau_{1}}$ such that $\|f\| \leq 1$, $\operatorname{coz}(f) \subset U$ and $\left|\delta_{s} T(f)\right|>R$.

Proof. (a) Let $g$ be an element in $C\left(K_{1}\right)^{\tau_{1}}$ such that $\|g\| \leq 1$ and $\left|\delta_{s} T(g)\right|>$ $\left\|\delta_{s} T\right\|-\varepsilon$. Let $V \subseteq K_{1}$ be an open set satisfying $[t] \subset V \subseteq \bar{V} \subseteq U$. By Urysohn's lemma there exists $0 \leq u \leq 1$ in $C\left(K_{1}\right)^{\tau_{1}}$ with $\left.u\right|_{\bar{V}} \equiv 1$ and $\left.u\right|_{K_{1} \backslash U} \equiv 0$. We observe that $t \notin \overline{\operatorname{coz}(1-u)}$, and thus, Lemma 3.3 implies that $\delta_{s} T(g(1-u))=0$. We therefore have

$$
\delta_{s} T(g)=\delta_{s} T(g(1-u)+g u)=\delta_{s} T(g u),
$$

which gives the desired statement for $f=g u \in C\left(K_{1}\right)^{\tau_{1}}$ with $\|g u\| \leq 1$ and $\operatorname{coz}(g u) \subset U$.

The proof of $(b)$ is very similar.

Lemma 3.7. Under the above assumptions, let $\left(t_{n}\right)$ and $\left(s_{n}\right)$ be sequences in $K_{1}$ and $K_{2}$, respectively, such that $\left[t_{n}\right] \neq\left[t_{m}\right]$, for every $n \neq m$ and $\left[t_{n}\right]=\operatorname{supp}\left(\delta_{s_{n}} T\right)$ for every $n \in \mathbb{N}$. Then $\sup \left\{\left\|\delta_{s_{n}} T\right\|: n \in \mathbb{N}\right\}<\infty$.

Proof. Arguing by contradiction, we assume that, $\sup \left\{\left\|\delta_{s_{n}} T\right\|: n \in \mathbb{N}\right\}=\infty$. Up to an appropriate subsequence, we can find a sequence $\left(U_{n}\right)$ of mutually disjoint open subsets in $K_{1}$ such that $\left[t_{n}\right] \subset U_{n}=\sigma_{1}\left(U_{n}\right)$ and $\sup \left\{\left\|\delta_{s_{n}} T\right\|: n \in \mathbb{N}\right\}=\infty$.

Since $\sup \left\{\left\|\delta_{s_{n}} T\right\|: n \in \mathbb{N}\right\}=\infty$, we apply Lemma 3.6 to find a subsequence $\left(n_{k}\right)$ and sequence $\left(f_{k}\right)_{k} \subset C\left(K_{1}\right)^{\tau_{1}}$ such that $\left\|f_{k}\right\| \leq 1, \operatorname{coz}\left(f_{k}\right) \subset U_{n_{k}}$ and $\left|\delta_{s_{n_{k}}} T\left(f_{k}\right)\right|>2^{k}$, for every natural $k$. The functions in the sequence $\left(f_{k}\right)_{k}$ are mutually orthogonal, therefore $f_{0}=\sum_{k=1}^{\infty} \frac{1}{k} f_{k}$ defines an element in $C\left(K_{1}\right)^{\tau_{1}}$. By orthogonality, for each $k_{0} \in \mathbb{N}$, we have

$$
\left|\delta_{s_{n_{k_{0}}}} T\left(f_{0}\right)\right|=\left|\delta_{s_{n_{k_{0}}}} T\left(\frac{1}{k_{0}} f_{k_{0}}+\sum_{k=1, k \neq k_{0}}^{\infty} \frac{1}{k} f_{k}\right)\right|=\left|\delta_{s_{n_{k_{0}}}} T\left(\frac{1}{k_{0}} f_{k_{0}}\right)\right|>\frac{2^{k_{0}}}{k_{0}},
$$

which is impossible.

Following a similar notation to that employed in [3], we set $Z_{1}:=\left\{s \in K_{2}\right.$ : $\delta_{s} T$ is bounded $\}$ and $Z_{2}:=\left\{s \in K_{2}: \delta_{s} T\right.$ is unbounded $\}$. Clearly $\sigma_{2}\left(Z_{i}\right)=Z_{i}$, for every $i=1,2$. The following conclusions can be straightforwardly derived from the previous results.

Corollary 3.8. (a) The set $\mathcal{S} u p p\left(Z_{2}\right):=\bigcup_{s \in Z_{2}} \operatorname{supp}\left(\delta_{s} T\right) \subseteq K_{1}$ is finite and satisfies $\sigma_{1}\left(\mathcal{S} u p p\left(Z_{2}\right)\right)=\mathcal{S}$ upp $\left(Z_{2}\right)$. Furthermore, every point in $\mathcal{S}$ upp $\left(Z_{2}\right)$ is non-isolated in $K_{1}$;

(b) The set $\left\{\left\|\delta_{s} T\right\|: s \in Z_{1}, \sigma_{2}(s) \neq s\right\}$ is bounded. 
Proof. (a) The first statement follows from Lemma 3.7. Suppose $t_{0} \in \mathcal{S} \operatorname{upp}\left(Z_{2}\right)$ is isolated in $K_{1}$. We know that $t_{0} \in \operatorname{supp}\left(\delta_{s} T\right)$ for certain $s \in Z_{2}$. In this case, we do not need the continuity assumptions in Proposition 3.4 to get a similar conclusion. Indeed, since $\left\{t_{0}\right\}$ is a closed subset, a function $f \in C\left(K_{1}\right)^{\tau_{1}}$ vanishes at $t_{0}$ if and only if $t_{0} \notin \overline{\operatorname{coz}(f)}$. Moreover, $g_{0}=\delta_{t_{0}}+\delta_{\sigma_{1}\left(t_{0}\right)} \in C\left(K_{1}\right)^{\tau_{1}}$ and $f-f\left(t_{0}\right) g_{0}$ vanished at $t_{0}$, for every $f \in C\left(K_{1}\right)^{\tau_{1}}$. Lemma 3.3 implies that $\delta_{s} T\left(f-f\left(t_{0}\right) g_{0}\right)=0$, and hence $\delta_{s} T(f)=\delta_{s} T\left(f\left(t_{0}\right) g_{0}\right)$ for every $f \in C\left(K_{1}\right)^{\tau_{1}}$. We note that the restriction of the real linear mapping $\delta_{s} T$ to the two dimensional subspace $\mathbb{C}_{\mathbb{R}} g_{0}$ is clearly continuous, and the same property holds for the linear mapping $\delta_{t_{0}} g_{0}: C\left(K_{1}\right)^{\tau_{1}} \rightarrow$ $\mathbb{C} g_{0}, f \mapsto \delta_{t_{0}}(f) g_{0}$. Therefore, the composed mapping $f \mapsto \delta_{s} T\left(f\left(t_{0}\right) g_{0}\right)=\delta_{s} T(f)$ is linear and continuous, which contradicts that $s \in Z_{2}$.

(b) Arguing by contradiction, we assume that $\left\{\left\|\delta_{s} T\right\|: s \in Z_{1}, \sigma_{2}(s) \neq s\right\}$ is unbounded. We can find a sequence $\left(s_{n}\right) \subset Z_{1}$ such that $2^{n} \leq\left\|\delta_{s_{n}} T\right\| \supsetneqq\left\|\delta_{s_{n+1}} T\right\|$. Clearly, $s_{n} \neq s_{m}$ and $s_{n} \neq \sigma_{2}\left(s_{m}\right)$, for every $n \neq m$ (we recall that $\left\|\delta_{s} T\right\|=$ $\left.\left\|\delta_{\sigma_{2}(s)} T\right\|\right)$. Applying Corollary 3.5(b) we deduce that $\operatorname{supp}\left(\delta_{s_{n}} T\right) \cap \operatorname{supp}\left(\delta_{s_{m}} T\right)=\emptyset$. Finally, Lemma 3.7 gives the desired contradiction.

Let $\Delta_{1}:=\left\{(s, t): s \in Z_{1}, t \in \operatorname{supp}\left(\delta_{s} T\right)\right\}$. We define a mapping $\vartheta: \Delta_{1} \rightarrow \mathbb{C}$ given by $\vartheta(s, t)=0$ if $\sigma_{1}(t)=t$ and $\vartheta(s, t)=\mu_{s}$ if $\sigma_{1}(t) \neq t$, where $\mu_{s}$ is the unique element given by Proposition 3.4.

Proposition 3.9. (a) Let $(s, t) \in \Delta_{1}$ with $\sigma_{1}(t) \neq t$, and let $g$ be any element in $C\left(K_{1}\right) \tau_{1}$ satisfying $\left.g\right|_{U} \equiv i$ for some open neighborhood $U$ of $t$. Then $T(g)(s)=$ $\mu_{s}=\vartheta(s, t)$.

(b) The function $\vartheta$ is bounded;

(c) The set $\left\{\left\|\delta_{s} T\right\|: s \in Z_{1}\right\}$ is bounded;

(d) $Z_{1}$ is closed.

Proof. (a) Follows straightforwardly from Proposition 3.4.

(b) If $\vartheta$ is unbounded, we can find a sequence $\left(s_{n}, t_{n}\right) \in \Delta_{1}$ satisfying $2^{n}<$ $\left|\vartheta\left(s_{n}, t_{n}\right)\right|<\left|\vartheta\left(s_{n+1}, t_{n+1}\right)\right|$. Obviously, $s_{n} \neq s_{m}$ and $s_{n} \neq \sigma_{2}\left(s_{m}\right)$, for every $n \neq m$ and $\operatorname{supp}\left(\delta_{s_{n}} T\right)=\left\{t_{n}, \sigma_{1}\left(t_{n}\right)\right\}$ with $t_{n} \neq \sigma_{1}\left(t_{n}\right)$. We claim that we can find a subsequence $\left(s_{n_{k}}, t_{n_{k}}\right) \subset \Delta_{1}$ satisfying $\operatorname{supp}\left(\delta_{s_{n_{k}}} T\right) \cap \operatorname{supp}\left(\delta_{s_{n_{m}}} T\right)=\emptyset$ for every $k \neq m$. Let us assume, on the contrary, that there exists a natural $n_{0}$ such that $\operatorname{supp}\left(\delta_{s_{n}} T\right)=\left\{t_{0}, \sigma_{1}\left(t_{0}\right)\right\}$, for every $n \geq n_{0}$. Let $g$ be any element in $C\left(K_{1}\right) \tau_{1}$ satisfying $\left.g\right|_{U} \equiv i$ for some open neighborhood $U$ of $t_{0}$. It follows from $(a)$ that $2^{n}<\left|\vartheta\left(s_{n}, t_{n}\right)\right|=\left|T(g)\left(s_{n}\right)\right|$, for every $n \geq n_{0}$, which contradicts that $T(g) \in$ $C\left(K_{2}\right)^{\tau_{2}}$. Having in mind that $\left\|\delta_{s_{n_{k}}} T\right\| \geq\left|\vartheta\left(s_{n_{k}}, t_{n_{k}}\right)\right|$, the desired contradiction follows from Lemma 3.7.

(c) We set $M_{1}=\sup \left\{\left|\vartheta\left(s, t_{s}\right)\right|:\left(s, t_{s}\right) \in \Delta_{1}\right\}$. Let $s$ be an element in $Z_{1}$, and let $t_{s}$ be an element in $\operatorname{supp}\left(\delta_{s} T\right)$. By Proposition 3.4 the identity

$$
\delta_{s} T(f)=T(1)(s) \Re \mathrm{e} \delta_{t_{s}}(f)+\vartheta\left(s, t_{s}\right) \Im \mathrm{m} \delta_{t_{s}}(f),
$$

holds for every $f \in C\left(K_{1}\right)^{\tau_{1}}$. Therefore, $\left|\delta_{s} T(f)\right| \leq\|T(1)\|+M_{1}$ for every $f \in$ $C\left(K_{1}\right)^{\tau_{1}}$ with $\|f\| \leq 1$.

(d) Let $M=\sup \left\{\left\|\delta_{s} T\right\|: s \in Z_{1}\right\}$. Fix an arbitrary $z_{0} \in \overline{Z_{1}}$ and a function $f \in C\left(K_{1}\right)^{\tau_{1}}$ with $\|f\| \leq 1$. We can find a net $\left(z_{\mu}\right) \subset Z_{1}$ converging to $z_{0}$ in the topology of $K_{2}$. Since $T(f)$ is a continuous function, we have $\left|T(f)\left(z_{\mu}\right)\right| \rightarrow$ $\left|T(f)\left(z_{0}\right)\right|$. On the other hand, by $(d)$ we have $\left|T(f)\left(z_{\mu}\right)\right|=\leq M$, for every $\mu$. 
Therefore, $\left|\delta_{z_{0}} T(f)\right|=\left|T(f)\left(z_{0}\right)\right| \leq M$, for every $f \in C\left(K_{1}\right)^{\tau_{1}}$ with $\|f\| \leq 1$, which proves that $\delta_{z_{0}} T$ is bounded, and hence $z_{0} \in Z_{1}$.

We state next the main result of this note, which provides an antidote to fill the gap we commented at the introduction.

Theorem 3.10. Every orthogonality preserving linear bijection between commutative unital real $C^{*}$-algebras is continuous.

Proof. Let $T: C\left(K_{1}\right)^{\tau_{1}} \rightarrow C\left(K_{2}\right)^{\tau_{2}}$ be an orthogonality preserving linear bijection. We shall prove that $Z_{2}:=\left\{s \in K_{2}: \delta_{s} T\right.$ is unbounded $\}=\emptyset$. Suppose, on the contrary, that $Z_{2} \neq \emptyset$. Since the set $Z_{1}$ is closed (see Proposition 3.9(d)) the set $Z_{2}$ is an non-empty open subset of $K_{2}$ with $\sigma_{2}\left(Z_{2}\right)=Z_{2}$. Find, via Urysohn's lemma, a non-zero function $g \in C\left(K_{2}\right)^{\tau_{2}}$ with $\operatorname{coz}(g) \subseteq Z_{2}$. By the surjectivity of $T$ there exists $0 \neq h \in C\left(K_{1}\right)^{\tau_{1}}$ satisfying $T(h)=g$. According to the notation above, we set $\operatorname{Supp}\left(K_{2}\right)=\bigcup_{s \in K_{2}} \operatorname{supp}\left(\delta_{s} T\right), \mathcal{S} u p p\left(Z_{1}\right)=\bigcup_{s \in Z_{1}} \operatorname{supp}\left(\delta_{s} T\right)$, and $\mathcal{S} u p p\left(Z_{2}\right)=$ $\bigcup_{s \in Z_{2}} \operatorname{supp}\left(\delta_{s} T\right)$. We claim that

$$
h(t)=0, \text { for every } t \in \mathcal{S} \operatorname{upp}\left(Z_{1}\right) .
$$

To prove the claim, let $t_{0}$ be an element in $\operatorname{Supp}\left(Z_{1}\right)$. Since $\mathcal{S} u p p\left(Z_{2}\right)$ is a $\sigma_{1}$ symmetric finite set in $K_{1}$ disjoint from $\mathcal{S} \operatorname{upp}\left(Z_{1}\right)$, there exist disjoint open sets $U_{1}, U_{2} \subseteq K_{1}$ and a function $k \in C\left(K_{1}\right)^{\tau_{1}}$ such that $\sigma_{1}\left(U_{j}\right)=U_{j}$ for every $j=1,2$, $t_{0} \in U_{1}, \mathcal{S} \operatorname{upp}\left(Z_{2}\right) \subseteq U_{2}, k^{*}=k$ (i.e. $\left.k\left(K_{1}\right) \subseteq \mathbb{R}\right), k\left(t_{0}\right)=1$, and $\operatorname{coz}(k) \subseteq U_{1}$. We claim that $T(k h)=0$. Indeed, for $s \in Z_{2}$, since $\mathcal{S} \operatorname{upp}\left(Z_{2}\right) \cap \overline{\operatorname{coz}(k)}=\emptyset$, Lemma 3.3 implies that $T(k h)(s)=\delta_{s} T(k h)=0$. For each $s \in Z_{1}$, and $t_{s} \in \operatorname{supp}\left(\delta_{s} T\right), k\left(t_{s}\right) \in$ $\mathbb{R}$ and hence $k h, k\left(t_{s}\right) h \in C\left(K_{1}\right)^{\tau_{1}}$ with $(k h)\left(t_{s}\right)=\left(k\left(t_{s}\right) h\right)\left(t_{s}\right)$. By Proposition 3.4 we also have:

$$
\begin{gathered}
\delta_{s} T(k h)=T(1)(s) \Re \mathrm{e} \delta_{t_{s}}(k h)+\vartheta\left(s, t_{s}\right) \Im \mathrm{m} \delta_{t_{s}}(k h), \\
=T(1)(s) \Re \mathrm{e} \delta_{t_{s}}\left(k\left(t_{s}\right) h\right)+\vartheta\left(s, t_{s}\right) \Im \mathrm{m} \delta_{t_{s}}\left(k\left(t_{s}\right) h\right) \\
=\delta_{s} T\left(k\left(t_{s}\right) h\right)=k\left(t_{s}\right) \delta_{s} T(h)=k\left(t_{s}\right) \delta_{s}(g)=0,
\end{gathered}
$$

because $s \in Z_{1}$ and $\operatorname{coz}(g) \subseteq Z_{2}$. This proves the second claim, and hence $T(k h)=$ 0 . The injectivity of $T$ implies $k h=0$ and hence $0=(k h)\left(t_{0}\right)=h\left(t_{0}\right)$, which completes the proof of the first claim.

By Lemma 3.3 the set $\mathcal{S} \operatorname{upp}\left(K_{2}\right)$ is dense in $K_{1}$. The subset $\mathcal{S} \operatorname{upp}\left(Z_{2}\right)$ is finite and every point in $\mathcal{S} \operatorname{upp}\left(Z_{2}\right)$ is non-isolated in $K_{1}$ (compare Corollary 3.8(a)). Since $\mathcal{S} \operatorname{upp}\left(K_{2}\right)=\mathcal{S} \operatorname{upp}\left(Z_{2}\right) \stackrel{\circ}{\cup} \operatorname{Supp}\left(Z_{1}\right)$. It is not hard to see, from the normality of $K_{1}$, that $\mathcal{S} \operatorname{upp}\left(Z_{1}\right)$ must be also dense in $K_{1}$. Since, by $(12), h(t)=0$ for every $t \in \mathcal{S} \operatorname{upp}\left(Z_{1}\right)$, we deduce from the continuity of $h$ that $h=0$, and hence $0=T(h)=g$, which gives the final contradiction.

A generalized version of [1, Corollary 3.10] for real $\mathrm{C}^{*}$-algebras read as follows:

Corollary 3.11. The following statements are equivalent:

(a) There exists a bi-orthogonality preserving linear bijection $T: C\left(K_{1}\right)^{\tau_{1}} \rightarrow C\left(K_{2}\right)^{\tau_{2}}$;

(b) There exists a real $C^{*}$-isomorphism $S: C\left(K_{1}\right)^{\tau_{1}} \rightarrow C\left(K_{2}\right)^{\tau_{2}}$;

(c) There exists a $C^{*}$-isomorphism $\widetilde{S}: C\left(K_{1}\right) \rightarrow C\left(K_{2}\right)$;

(d) $K_{1}$ and $K_{2}$ are homeomorphic. 


\section{REFERENCES}

[1] J. Garcés and A. M. Peralta, Orthogonal forms and orthogonality preservers on real function algebras, Linear and Multilinear Algebra 62, no. 3, 275-296 (2014). DOI:10.1080/03081087.2013.772998

[2] S. Goldstein, Stationarity of operator algebras, J. Funct. Anal. 118, no. 2, 275-308 (1993).

[3] K. Jarosz, Automatic continuity of separating linear isomorphisms, Canad. Math. Bull. 33, no. 2, 139-144 (1990)

[4] B.R. Li, Real operator algebras, World Scientific Publishing (Singapore), 2003.

Departamento de Análisis Matemático, Facultad de Ciencias, Universidad de Granada, 18071 Granada, Spain.

E-mail address: aperalta@ugr.es 